\title{
Les diverses formes pour la compensation dans la protection juridique de l'environnement: un défi pour l'épistémologie juridique
}

Fecha de recepción: 16 de noviembre de 2012

Fecha de aceptación: 22 de noviembre de 2013

Doi: dx.doi.org/10.12804/acdi7.2014.05

\section{Gabriela G B Lima*}

Résumé: La compensation est un outil d'adaptation entre différents intérêts. Par rapport à l'environnement, il existe une diversité de formes qui appliquent de façon différente l'une de l'autre, la technique de la compensation. Autrement dit, des situations juridiques différentes appliquent la compensation comme une façon de régler un enjeu: on peut la trouver dans la forme d'indemnisation, comme dans de cas de la Fonderie du Trail et la Commission de Compensation des Nations Unies; de réglementation d'un dommage futur, étant une compensation ex ante facto, comme dans le cas de la Convention de Ramsar; et par des voies juridiques qui incitent le comportement privé à travers l'usage de certains outils de marché, comme les Mécanismes de Dèveloppement Propre, ou dans le domaine du droit national, la compensation de la biodiversité française, la «mitigation banking» américaine, la «Servidão Ambiental» Brésilienne, et l'écocompensation chinoise, entre autres. Le défi épistémologique se présente dans la diversité de sources, ainsi que d'acteurs et de domaines d'action dans le cadre juridique

* Doctorat en Droit du Centre Universitaire de Brasília, cotutelle avec l'Université Aix-Marseille III, 2012.E-mail: gblima@gmail.com

Para citar este artículo: G B Lima, Gabriela (2014), "Les diverses formes pour la compensation dans la protection juridique de l'environnement: un défi pour l'épistémologie juridique”. ACDI - Anuario Colombiano de Derecho Internacional vol. 7, pp. 161-188. doi: dx.doi.org/10.12804/acdi7.2014.05 
environnemental, en y exigeant un élargissement de la vision du droit étatique. Il s'agit aussi d'intégrer une interprétation systémique pour le rapport entre les systèmes juridiques, écologiques et économiques concernés. Il est possible d'utiliser quelques outils comme le pluralisme juridique, la théorie des systèmes et l'analyse économique du droit de l'environnement. Les concepts de corégulation et autorégulation peuvent aussi aider dans cet élargissement. D’ailleurs, des limites sont nécessaires pour l'équilibre entre la mise en oeuvre des intérêts écologiques et économiques. Ces limites sont données par le droit, par l'interprétation systémique, par l'État et par un renforcement de la responsabilité des entités privées. L'analyse présente d'abord les caractéristiques de la compensation dans des instruments économiques et juridiques. Ensuite, il est vérifié comment le pluralisme juridique, l'interprétation systémique, l'analyse économique et les concepts de corégulation et autorégulation peuvent-ils être utiles pour le regard épistémologique de la compensation, ainsi que pour son étude juridique.

Most Clés: Environnement, compensation, interprétation systémique, corégulation, autorégulation, cadre juridique environnemental.

Los diferentes mecanismos de compensación en la protección jurídica del medio ambiente: un desafío para la epistemología jurídica

Resumen: La compensación es una herramienta de adaptación entre intereses diversos. Con relación al medio ambiente, existe una variedad de mecanismos que aplican de distintas formas la técnica de la compensación. Dicho de otra forma, diferentes situaciones jurídicas aplican la compensación como un mecanismo para solucionar un diferendo, puede darse bajo la perspectiva de la indemnización, como en el caso "Trail Smelter", así como en la Comisión de Compensación de Naciones Unidas; para reglamentar un daño futuro, siendo una compensación ex ante facto, como en el caso de la Convención Ramsar; por vías jurídicas que incitan al comportamiento privado a través del uso de ciertas herramientas del mercado, como mecanismos de desarrollo propio, o en el ámbito del derecho internacional, la compensación de la biodiversidad francesa, la "mitigation banking" estadounidense, la "Servidão Ambiental" Brasilera, y la ecocompensación china, entre otras. El desafío epistemológico se presenta como consecuencia de la variedad de fuentes, de la variedad de actores así como del campo de acción en el marco jurídico medioambiental, exigiendo una ampliación de la visión del derecho estatal. También se trata de integrar una interpretación sistémica 
para una interrelación entre los sistemas jurídicos, ecológicos y económicos concernidos. Es imposible acudir a algunas las herramientas como el pluralismo jurídico, la teoría de los sistemas y el análisis económico del derecho ambiental. Los conceptos de corregulación y autorregulación pueden ser útiles en la ampliación que se requiere. Entre otras, es necesario establecer límites para que haya un equilibrio entre la puesta en funcionamiento de los intereses ecológicos y los económicos. Estos límites los da el derecho por la vía de la interpretación sistémica, por el Estado y por el fortalecimiento de la responsabilidad de las entidades privadas. El análisis presenta, en primer lugar, las características de la compensación en los instrumentos económicos y jurídicos; en segundo lugar, se comprueba como el pluralismo jurídico, la interpretación sistémica, el análisis económico y los conceptos de corregulación y autorregulación pueden ser útiles para la mirada epistemológica de la compensación, así como para su análisis jurídico.

Palabras clave: Medio ambiente, compensación, interpretación sistémica, corregulación, autorregulación, marco jurídico ambiental.

Different compensation mechanisms in the judicial protection of the environment: A Challenge for Juridical Epistemology

Abstract: Compensation is an adaptive tool among diverse interests. In relation to the environment, a variety of mechanisms exist which apply distinct forms of compensation. Put another way, different juridical situations apply compensation as a means of resolving a difference. It can be from the perspective of indemnization, as in the Trail Smelter case or similarly in United Nations Compensation Commission; it can be to regulate future damage. It can be seen as a form of ex ante facto (before the fact) compensation as in the Ramsar Convention case. It can be a juridical route to induce private behaviour through the use of certain market tools as personal development mechanisms or within the ambit of international law as in France's biodiversity compensation, the United States" "mitigation banking", Brazil's "Servidão Ambiental" and China's eco-compensation, among others. The epistemological challenge presents itself as a consequence of the variety of sources, the variety of actors and likewise the fields of action within the environmental juridical framework. These demand a broadening of the vision of state law. It also demands an attempt to integrate a systemic interpretation of the juridical, ecological and economic systems concerned. It is impossible to resort to some tools such as juridical pluralism, systems theory and the 
economic analysis of the environment. The concepts of co-regulation and self-regulation could be useful in the broadening that is required. Among other things it is necessary to set limits so that there is an equilibrium point around which ecological and economic interests may function. These limits allow us to set the path towards a systemic interpretation for the State and for the strengthening of the responsibilities of private entities. This analysis presents, in the first place, the compensation characteristics found in economic and juridical instruments; in the second place it examines how juridical pluralism, systemic interpretation, economic analysis and the concepts of co-regulation and self-regulation could be useful for the epistemological view of compensation as well as for juridical analysis.

Key words: Environment, compensation, systemic analysis, co-regulation, self-regulation, environmental juridical framework.

\section{Introduction}

La compensation est un outil d'adaptation. En langage usuel, c'est équilibrer un effet souvent négatif par l'autre, neutraliser la perte par le gain. ${ }^{1}$ En Droit, la compensation est une technique de traitement des intérêts parfois contradictoires dans la création et l'application de normes. Quand il y a la nécessité d'intégrer différents intérêts, la compensation devient une des techniques applicables comme juste pour cette interaction. C'est dans ce sens général que nous allons la traiter ici.

Dans la protection juridique de l'environnement, la compensation peut être trouvée dans plusieurs exemples: dans la Convention de Ramsar, dans les mécanismes de développement propre, dans quelques arbitrages étatiques, dans les «marchés» de biodiversité en France, au Brésil, aux ÉtatsUnis, l'ecocompensation en Chine, entre autres. Ce sont des normes qui ont été faites par des acteurs publics ou/et privés, par contrat et/ou par des outils économiques, dans l'accomplissement d'une obligation ou/et volontairement et qui concernent différentes manières de l'interaction des enjeux écologiques et économiques dans les normes juridiques.

Cette hétérogénéité d'acteurs et de formes normatives pour la compensation est un défi pour son analyse juridique. Ils sont, en quelque sorte, des conséquences de la pluralité de la globalisation dans le droit: pluralité

1 Le Robert, 2011. 
des acteurs produisant des normes juridiques et pluralité des enjeux qui sont touchés par des normes juridiques.

Cette pluralité renforce l'émergence des concepts du pluralisme juridique, la corégulation et l'autorégulation, pour analyser la légitimité des acteurs et les sources des ordres juridiques différents de celles seulement étatiques. C'est aussi l'émergence de la prise en compte de l'analyse économique du droit de l'environnement, en permettant un rapport systémique de l'étude des normes, en face de l'interdisciplinarité qui, dans le cas de l'environnement, consiste surtout en l'interaction des enjeux économiques et écologiques en général.

Dans ce domaine, le droit est vu dans son concept le plus général, comme un instrument pour organiser des enjeux entre certaines parties. Ce qui peut être questionné, par ailleurs, c'est la nécessité de la contrainte étatique pour la caractérisation du droit, entre autres. Ce sont les questions liées non seulement à une crise du concept de droit étatique, mais aussi de l'État en tant qu'acteur majeur dans la conduction du droit en société.

Dans quelle mesure l'insuffisance de l'État est-elle assimilée par la protection juridique de l'environnement, lorsque celui-ci est un domaine juridique étatique par excellence? Comment la protection de l'environnement est-elle encadrée par un contexte de pluralisme juridique? De forme plus précise: comment encadrer les différentes sources publiques et privées en droit de l'environnement? Comment améliorer l'équilibre dans l'interaction des divers enjeux économiques et écologiques touchés par les normes dans un cadre pluraliste?

Ces enjeux seront analysés par certains exemples de compensation dans la protection juridique de l'environnement. Lorsque la variété d'exemples est si évidente qu'ils peuvent être analysés par activité, par problème, par pays, etc., il est difficile de choisir une forme d'analyse sans exclure d'autres. Toutefois, puisque nous sommes intéressés à explorer certains des effets de pluralité dans le monde juridique, l'analyse prendra juste quelquesuns des exemples pour étudier la pluralité d'acteurs des normes juridiques.

Parmi les exemples se trouvent l'arbitrage étatique «le Train Smelten», la Commission de Compensation des Nations Unies et certaines des règles de compensation comme dans la Convention de Ramsar et les directives 2001/42/CE, 85/337/CEE, 92/43/CEE de l'Europe, entre autres, en illustrant l'approche juridique «traditionnelle», c'est-à-dire, dans le domaine plutôt étatique d'action, en concernant surtout la définition des droits et devoirs. Ils encadrent des formes indemnitaires, préventives d'un litige étatique et la compensation d'un dommage. 
De plus, dans la recherche d'une meilleure effectivité juridique, les interactions entre certains instruments économiques ${ }^{2}$ et des outils juridiques ont émergé, dont certains mécanismes de compensation. Ce sont des formes incitatives, pour des changements des comportements économiques. Ces mécanismes peuvent concerner la gestion d'une pollution; d'ailleurs, ils peuvent encadrer le financement pour des objectifs de développement durable en général et, d'une forme plus précise, ils peuvent encadrer des paiements pour des restaurations et des conservations de la nature, par exemple, des paiements pour des services environnementaux et des «marchés» de biodiversité.

Ces mécanismes de compensation sont ici illustrés par des mécanismes de développement propre du Protocole de Kyoto, des projets de Rédutions d'émissions liées au déboisement et à la dégradation des forêts (REDD) existantes, des «mitigating banking» américain, la CDC Biodiversité française, la «servitude forestière» brésilienne et l'«écocompensation» chinoise.

Cette interaction avec les outils économiques permet différentes participations publiques et privées au sein de la constitution et l'application des normes pour l'environnement, et peut être une meilleure interaction entre les enjeux économiques et écologiques dans la norme juridique. D'ailleurs, ces cas montrent que l'État et le droit étatique sont encore importants pour imposer des limites dans cette interaction, pour l'effectivité de la protection de l'environnement. Pour tout ce contexte, l'interaction entre les outils juridiques et économiques a un rôle important.

Ainsi, d'abord, il faut revenir dans l'analyse des exemples de différents encadrements juridiques pour la compensation (1). Ils permettent de poser des questions de pluralité d'acteurs et de pluralité d'enjeux touchés par les normes dans un contexte de pluralisme juridique (2). C'est dans ces questions de pluralité que les concepts de pluralisme juridique, corégulation, autorégulation, ainsi que de l'analyse économique du droit de l'environnement et son rapport systémique, sont compris. Tout ce contexte permet de vérifier quelques-unes des limites et possibilités pour l'épistémologie juridique en face de ces exemples de compensation pour l'environnement.

2 Nous considérons le concept d'outil économique de l'Organisation de Coopération et de Développement Économiques (OCDE). Apliquée à l'environnement, ce sont des mesures incitatives et économiques comme des droits, taxes, paiements des services environnementaux, des fonds, création de marchés, entre autres. Parmi d'autres, voir les Recommandations C(72)128 de 26 mai 1972, (C(90)177/FINAL), et la Recommandation du Conseil de l' OCDE le 21 avril 2004. 


\section{Les différentes formes juridiques pour la compensation}

La compensation dans la protection juridique de l'environnement peut être faite de différentes manières. D'abord, il est important de comprendre les exemples de compensation dans une approche juridique étatique traditionnelle (1.1). Ensuite, l'analyse abordera les exemples de mécanismes de compensation en intégrant des outils économiques (1.2). Il convient d'expliquer qu'il ne consiste pas dans un approfondissement analytique des difficultés possibles pour la mise en oeuvre de chaque exemple. Ils sont abordés ici pour avoir un regard panoramique de quelques formes juridiques possibles pour la compensation et pouvoir, après, étudier quelques enjeux de cette pluralité de formes dans la protection de l'environnement.

\subsection{La compensation dans des outils juridiques «traditionnels» pour l'environnement}

Dans ce domaine de la compensation dans des outils juridiques traditionnels, centralisés dans le domaine étatique d'action. Il y est possible de voir brièvement une variation de la compensation dans des formes juridiques d'indemnisations pour des dommages environnementaux, de technique de prévention d'un litige étatique, et de réglementation ex ante facto d'un dommage à fin de rationaliser l'intervention humaine dans la nature.

Comme une indemnisation pour des dommages environnementaux, il est erroné de dire que toute indemnisation sera une compensation, lorsqu'elle n'est pas toujours prévue dans l'exact mot «compensation». ${ }^{3}$ D'ailleurs, il en existe quelques exemples où la compensation en est l'indemnisation d'un dommage environnemental. Nous pouvons la trouver dans les arbitrages étatiques comme le «The Trail Smelten», où un problème de pollution transfrontalier a été abordé. ${ }^{4}$ La compensation ici concerne l'indemnisation des

3 Par exemple, il n'est prévu dans la Convention de Bâle sur le contrôle des mouvements transfrontaliers de déchets dangereux, entre autres exemples. Dans ce cas, il n'est indiqué que le mot «indemnisation» pour la responsabilité environnementale.

4 Cet arbitrage a été donné pour un problème de pollution de l'air venu d'une entreprise du Canada, affectant quelques propriétés des États Unis qui ont réclamé une compensation. En 1928, une Commission Internationale Conjointe pour l'investigation a été constituée, et a donné une recommandation en stipulant l'indemnisation de $\$ 350$, 000 pour les dommages, en 1931. Néanmoins, les dommages ont continué et en 1935 les parties ont signé une 
effets de pollution subis par les parties et représente une responsabilité environnementale internationale pour des dommages environnementaux.

Un autre exemple est la Commission de la Compensation des Nations Unies, subsidiaires du Conseil de Sécurité. Établie en 1991, comme un organisme administratif ${ }^{5}$ pour la mise en oeuvre de la responsabilité de l'Irak par rapport aux dommages environnementaux ${ }^{6}$ conséquents de l'invasion du Koweït en 1990. L'Irak a accepté sa légitimité devant le Conseil de Sécurité et toute indemnisation devrait être payée après un processus d'appréciation des faits et de l'évaluation des coûts, suivi par cette Commission. Le paiement était retiré d'un Fonds. ${ }^{7}$ Depuis 1991, plus de 2.6 millions réclamations des individus, corporations et États ont été faits jusqu'à 2007. ${ }^{8}$ La Commission a été beaucoup critiquée, ${ }^{9}$ toutefois, sa structure c'est une avance pour la responsabilité internationale des dommages environnementaux.

Convention pour l'établissement d'un arbitrage. En 1938, une décision a stipulé l'indemnisation de $\$ 78.000$, (pour le dommage entre 1932 et 1937), entre autres mesures. D'ailleurs, parce que la pollution continuait, le cas n'était fini que dans l'arbitrage de 1941.

5 La Comission était structurée par comités, or panels de 3 commissaires et experts indépendants pour l'évaluation de ces faits, coûts, etc. Il y avait 19 panels, dont le «F4» était pour les dommages environnementaux. Sur la nature de la Commission: Report $\mathrm{f}$ the UM Secretary-General S/22559,1991, paragraph 20, elle n'est pas une courts ni un tribunal arbitral, mais juste un organisme administrative pour la mise en oeuvre de la responsabilité de l'Irak. The UNCC Secretariat. Introduction. Disponible en: http://www.uncc.ch/introduc.htm. Acéssé en 29.12.2011

6 Selon la décision n. 7, paragraphe 35 du Conseil de la Commission: "These payments are available with respect to direct environmental damage and the depletion of natural resources as a result of Iraq's unlawful invasion and occupation of Kuwait. This will include losses or expenses resulting from: (a) Abatement and prevention of environmental damage, including expenses directly relating to fighting oil fires and stemming the flow of oil in coastal and international waters; (b) Reasonable measures already taken to clean and restore the environment or future measures which can be documented as reasonably necessary to clan and restore the environment; (c) Reasonable monitoring and assessment of the environmental damage for the purposes of evaluating and abating the harm and restoring the environment; (d) Reasonable monitoring of public health and performing medical screenings for the purposes of investigation and combating increased health riskes as a result of the environmental damage; Depletion of or damage to natural resources". The UNCC Secretariat. Décision 07. Disponible en: http://www.uncc.ch/decision/dec_07r.pdf. Acessé en 22.12.2011.

7 Fonds créé par la Résolution 692 (2001). Ce fonds a été formé par un pourcentage de la vente de pétrole de l'Irak. La Commission était responsable aussi pour son administration.

8 Les réclamations étaient en catégories pour les individus, pour des corporations et pour des gouvernements et organisations internationales. The UnCC Secretariat. The Claims. Disponible en: http://www.uncc.ch/theclaims.htm. Acesse en 12.12.2011.

9 Des critiques concernaient la légitimité de la création de la Commission faite par le 
Dans la forme d'une indemnisation, la compensation est utilisé comme une façon d'extinction d'une obligation étatique d'un État qui a causé des dommages à un autre État. Son application est justifié à partir de la constatation d'une responsabilité environnementale internationale. Dans ce sens, elle est forcement lié à la due diligence ${ }^{10}$ des États dans leurs comportements. Lorsqu'un dommage est constaté, la responsabilité est engagée si l'État n'a pas pris des mesures préventives telles que la notification des effets d'une activité transfrontière à l'État concerné, ou la prendre en compte d'actions nationales pour éviter et pour minimiser les dommages de cette activité, entre autres situations.

Au sein de la due diligence de l'État, la compensation peut prendre une fonction distincte de l'indemnisation du dommage: elle peut concerner une façon d'éviter l'occurrence d'une responsabilité environnementale internationale. Il consiste la situation où la compensation prendre la forme d'une sorte de principe général de prévention de litiges. ${ }^{11}$ Par exemple, selon la Résolution de 1961 sur l'Utilisation des eaux non maritimes internationales de l'Institut de Droit International dans sa session à Salzburg, un État ne pouvait pas utiliser une ressource naturelle en préjugeant la possibilité d'usage par un autre État sans une compensation, ${ }^{12}$ étant aussi l'interprétation de la

Conseil de Sécurité; sur la manque de transparence dans l'appréciation des experts, sur le contradictoire, entre autres. Martin, Jean-Christophe. The United Nations Compensation Commission Practice with regards to environmental claims. In: Majean-Dubois, Sandrine. The transphormations of international environmental law, 2011, p. 263.

10 Étant, en droit international, un principe général du droit, la notion de la due diligence renvoie à une obligation de comportement de la part d'un sujet de droit, l'État, à fin de contrôler les conséquences de leurs actions à d'autres États. Ce sont, par exemple, les obligations de prévention que sont attendues en droit international, tels que la notification d'une activité ayant des impacts transfrontaliers, ou l'internalisation d'un traité environnemental signé, ainsi que des voies pour son accomplissement. Koivurova, Timo, «Due Diligence», Max Planck Encyclopedia of Public International Law, disponible sur http://www.arcticcentre.org/ loader.aspx?id=78182718-d0c9-4833-97b3-b69299e2f127; Voir aussi: Sadeleer, Nicolas de, "Comentários sobre o status no direito internacional de três princípios ambientais", in: Varella, Marcelo Dias; Barros-Platiau, Ana Flávia (orgs), "Proteção internacional do meio ambiente", UNITAR, UniCEUB e UnB, 2009, p. 46.

11 Bilder, Richard. Settlement of Disputes in Field of International Law of Environment. The Hague Academy of International Law, p. 222-225.

12 Article 4, Résolution de l'Institut de Droit International, de la Session de Salzbourg 1961, Utilisation des eaux internationales non maritimes. Disponible en: http://www.idi-iil. org/idiF/resolutionsF/1961_salz_01_fr.pdf Acessé en 20.12.2011. 
Convention pour l'usage de l'eau internationale, en 1997, en New York. ${ }^{13}$ Aussi de forme similaire, dans la Déclaration de Stockholm de 1972, principe 22. C'est aussi le cas de la Convention de Ramsar, de 1971, en vigueur en 1975, entre autres.

Concernant la forme de réglementation d'un dommage, il consiste une situation juridique distincte tant de l'indemnisation d'un dommage en raison d'une responsabilité environnementale, que de l'usage de la compensation pour prévenir cette responsabilité. La réglementation d'un dommage utilise la compensation pour rationaliser l'action humaine dans la nature. $\mathrm{La}$ Convention de Ramsar peut aussi en être un exemple. Sa règle de compensation envisage de faire rationaliser l'intervention humaine dans la nature, étant une compensation ex ante facto, c'est-à-dire, avant le l'occurrence du dommage. Dans ce cas elle se différencie de la situation juridique de responsabilité environnementale, qui dans la forme d'une compensation expost facto (après le dommage), englobe la compensation comme une indemnisation.

Considérant la Convention de Ramsar, selon son article 4.2, une compensation doit exister dans le cas où l'intérêt national est formé et l'intervention dans la nature est inévitable. Elle concerne la dernière étape, après les étapes d'éviter, minimiser et compenser un dommage. Son application suit le principe «pas de perte nette» ${ }^{14}$ et son caractère d'additionnalité: la compensation doit produire des effets positifs supérieurs aux pertes. L'atteinte à l'environnement doit prendre place ensemble avec une mesure compensatoire pour maintenir les fonctions écologiques qui seront perdues à l'occasion de cette atteinte.

Cette application en trois étapes et le principe «pas de perte nette» sont encadrés comme une logique générale pour la compensation des dommages environnementaux. Ces caractéristiques sont venues du contexte américain, surtout avec le «Clean Water Act». ${ }^{15}$ C'est aussi la logique des directives 2001/42/CE, pour l'évaluation des incidences des plans et programmes, et

13 Article 7 (2). Convention on the Law of the Non-navigational Uses of International Watercourses1. New York, 21 May 1997.

14 En anglais, "No Net Loss" - A target for a development project in which the impacts on biodiversity (...) are balanced or outweighed by measures taken to avoid and minimize the project's impacts, (...), so that no loss remains. Madsen, Becca; Carroll, Nathaniel; Moore Brands, Kelly; 2010. State of Biodiversity Markets Report: Offset and Compensation Programs Worldwide. Disponible en: «http://www.ecosystemmarketplace. com/documents/ acrobat/sbdmr.pdf», p. 2. Accésé en 02.03.2012.

15 7a Session de la Conférence des Parties, San José, Costa Rica, de 10 au 18 mai 1999. 
la directive 85/337/CEE, pour l'évaluation des incidences de certains projets publics et privés sur l'environnement. Au titre de ces évaluations, le maître d'ouvrage doit fournir une description des mesures envisagées pour éviter, réduire ou compenser des effets négatifs. Des règles compensatoires existent aussi dans la Narute 2000 (directive 92/43/CEE).

Ce sont des mesures compensatoires comprises dans des études d'impact dans un processus d"évaluation des conséquences nocives des activités en société. Il concerne une mesure qui vise à compenser les pertes environnementales par la création de gains environnementaux similaires à la nature de ces pertes-ci. Il consiste dans une façon de rationalisation de l'action humaine dans le sens de prévenir les risques environnementaux à partir d'un regard plus technique de l'intervention, l'un qui exige toute une évaluation des effets de l'activité pour l'autoriser.

Ce sont juste quelques exemples de la compensation dans des formes juridiques «traditionnelles», où la compensation est présente dans des définitions de droits et devoirs dans le cadre étatique de création de normes. Il importe maintenant de vérifier des exemples de la compensation dans l'intégration entre les outils juridiques et économiques, qui est, en effet, un effort pour améliorer l'effectivité dans la protection juridique de l'environnement, et qui parfois peut encadrer une pluralité d'acteurs dans les sources juridiques.

\subsection{La compensation dans l'interaction entre des outils économiques et juridiques pour l'environnement}

La compensation dans l'interaction entre les outils économiques et juridiques sont des compensations qui varient entre des actions publiques et privées. Elles sont distinctes des compensations traditionnelles, car elles mettrent en évidence un droit flexible, ${ }^{16}$ car l'élément principal n'est pas seulement

Disponible en: http://www.ramsar.org/cda/fr/ramsar-documents-resol-resolutionvii-21-21534/main/ramsar/1-31-107\%5E21534_4000_1_. Acessé: 29.12.2011.

16 Pour cette recherche, la flexibilité du droit consiste dans la construction de la norme juridique par la proposition d'une incitation, et non seulement d'une obligation juridique. Il concern une façon de différencier cet approche innovatrice de la norme environnementale de la perspective plus traditionnelle de "command and control". Sadeller, Nicolas de, «Les approches volontaires en droit de l'environnement, expression d'un droit post-moderne ?") In: Hervé-Fournenreau, Nathalie, «Les approches volontaires et le droit de l'environnement», PUR, p. 45-47. 
l'obligation juridique, mais l'incitation du comportement privé, ${ }^{17}$ un droit hybride, car l'efficacité juridique intègre tant des actions publiques que privées dans la formation de réseaux juridiques. ${ }^{18}$ Nous allons voir brièvement des mécanismes de développement propre du Protocole de Kyoto, les REDDs, le «mitigating banking» aux États-Unis, la CDC Biodiversité française, le «marché de terres» brésilien, et l'écocompensation en Chine. L'importance de ces exemples c'est surtout dans les efforts publics et privés dans une meilleure interaction entre les enjeux économiques et écologiques.

Les outils économiques concernent ceux conceptualisés par l'OCDE, ${ }^{19}$ dont la création des marchés et les paiements pour les services environnementaux. Ces marchés ne sont pas compris comme des échanges sans l'État, mais l'utilisation de la logique de marché par l'État et, parfois par des acteurs privés dans l'encadrement d'échanges autour d' un enjeu environnemental. C'est une approche juridique pour inciter le changement du comportement des acteurs économiques.

Concernant le Protocole de Kyoto, nous pouvons voir l'encadrement de la participation privée dans la coordination internationale pour des objectifs de développement durable. Nous voulons détacher surtout le «mécanis-

17 Arkkainen, B.; Radley C., "Information-forcing environmental regulation", Florida State University Law Review, Vol. 33:861, 2006, p. 861.; voir aussi: Wyeth, George B., "'Standard" and "Alternative" Environmental Protection: The Changing Role of Environmental Agencies", William \& Mary Environmental Law and Policy Review Volume 31 | Issue 1, Article 3, 2006, disponible sur http://scholarship.law.wm.edu/wmelpr/vol31/iss1/3, p. 72.; Harrington, Winston; Morgenstern, Richard D., "Economic Incentives versus Command and Control, what's the best approach for solving environmental problems?”, Ressources, Fall/Winter 2004, disponible sur http://www.rff.org/rff/Documents/RFF_Resources_152_ecoincentives.pdf, p. 17.

$18 \mathrm{Il}$ consiste dans un droit hybride pour englober les sources publiques et privées du droit. Cafaggi, Fabrizio, «New Foundations of Transnational Private Regulation», Journal of Law and Society, Volume 38, Number 1, March, 2011, p. 20.; Ost, Françoise; Kerchove, E., "De la pyramide au reseau. Pour une théorie dialectique du droit», Bruxelles: Publications des Facultés universitaires Saint-Louis Boulevard du Jardin Botanique, 2002, p. 186; voir aussi: Teubner, Gunther, «Droit et réflexivité. L'auto-référence en droit et dans l'organisation»,L.G.D.J - Bruylant, 1996, p. 163.

19 Les outils économiques en matière environnementale ont été promus principalment par l'Organisation de Coopération et de développement économiques (OCDE) depuis 1972, par la Recommandation C(72)128 de 26 mai 1972. Ils concernent les mesures incitatives e économiques tels que des taxes, des fonds, des créations de marchés, des permis pour l'use de ressources naturelles, des quotas, entre autres. Ce sont des instruments pour inciter l'utilisation durable des ressources naturelles. OCDE, Recommandation du Conseil sur l'utilisation des Instruments Économiques pour Faciliter la Conservation et l'exploitation durable de la biodiversité. Enteriné par les ministres de l'Environnement le 20 avril 2004 Adopté par le Conseil de l' OCDE le 21 avril 2004, p. 05. 
me pour un développement propre» (MDP). Il fait partie des mécanismes ${ }^{20}$ pour la mise en oeuvre du Protocole, qui sont des compensations diverses pour l'échange de droits définis autour des dommages environnementaux. Surement la mise en oeuvre du Protocole a ses propres difficultés, ${ }^{21}$ toutefois, il est un exemple de l'usage de la compensation pour inciter le changement des comportements des acteurs économiques dans des objectifs de développement durable.

Il est aussi possible de mentionner les REDDs, au sein de la Convention-Cadre des Nations unies sur les changements climatiques. En général, ce sont des mécanismes pour des paiements transnationaux pour des mesures de préservation des forêts, entre autres. L'engagement entre l'État et la société civile est important pour ce mécanisme. Des projets existent déjà par le Programme ONU-REDD, entre autres. ${ }^{22}$

Des exemples des États-Unis, France et Brésil ici utilisés sont des créations de crédits et d'actifs pour la restauration ou la préservation des espaces naturels en caractérisant des mécanismes de compensation par une logique de marché. Ces crédits peuvent être vendus par ceux qui ont une obligation de compenser leurs activités ou de forme volontaire. Les acteurs effectuant le travail de restauration peuvent être publics ou privés, selon l'exemple.

Aux États-Unis, il y a la mitigation de compensation pour l'accomplissement du «Clean Water Act», \404, pour les zones humides et des rivières. Les compensations touchent les conséquences résiduelles des activités, sous le critère de trois étapes et le principe «pas de perte nette». Les entreprises peuvent choisir de compenser elles-mêmes, par restauration ou préservation, ou acheter un crédit d'une banque de compensation («mitigation banking»), en achetant des crédits représentatifs d'actions de restauration. Il y a aussi un système de crédit pour des restaurations, le «National Recovery Credit System», le "Acres for America" programme volontaire dirigé par Walmart, le «Bureau of Land Management Offsite Mitigation Policy», entre autres. ${ }^{23}$

20 Les autres mécanismes sont un marché d'échange des quotas, qui est l'échange des «émissions licites» pour les pays de l'annexe I, et la «mise en œuvre conjointe» (MOC), qui permet aux pays de l'annexe I d'acheter des crédits d'émission en investissant dans des projets de réduction d'émission d'un autre pays de cette annexe.

21 Tabau, Anne Sophie. Les interactions des contrôles internationaux et communautaires de La mise en oeuvre du Protocole de Kyoto. Thèse pour l'obtention du diplôme de Doctorat de Droit Public. Université de Droit, D'Economie et de Sciences d'Aix-Marseille III.

22 Un-Redd Programme. Disponible en: «http:/ / www.un-redd.org» Accédé en 02.03.2012.

23 L'agence responsable pour la mise en oeuvre du «Clean Water Act» et les formes de 
En France, il existe la CDC Biodiversité, depuis 2008. C'est un opérateur financier qui a été créé à l'initiative de la Société Forestière de la Caisse des Dépôts, Société Anonyme. C'est une opération en concertation avec les pouvoirs publics, les grandes associations de protection de la nature et les autorités scientifiques. Ayant pour base le principe «pas de perte nette», ces services assurent la gestion des travaux de restauration de la nature en créant des crédits de biodiversité. Les résultats sont la création des réserves d'actifs naturels (RAN) susceptibles d'être reconnus comme des mesures compensatoires de projets d'aménagement du territoire. Il y a déjà quelques projets actifs comme en Camargue sur la plaine de la Crau, entre autres. ${ }^{24}$

Au Brésil, l'utilisation des mécanismes de compensation est tenue dans la régularisation des propriétés privées. Ils concernent des échanges d'actifs et passifs environnementaux encadrés dans des limites de la Réserve Légale (RL) et de l'Aire de Préservation Permanente (APP) du Code forestier. ${ }^{25}$ Les passifs sont des pourcentages de terres qui manquent dans ses propriétés pour compléter la quantité exigée par la RL et l'APP et des actifs concerne des pourcentages des propriétés qui dépassent le requis par la loi.

Les échanges sont faits entre particuliers, ayant le soutien de l'organisation non gouvernementale, la The Nature Conservancy (TNC), dans l'identification et l'échange de ces actifs, depuis plus de 10 ans ${ }^{26}$ Le

compensation c'est les «US Army Corps of Enginneers - US ACE». De plus, il y a environ 96 «mitigation banking actifs», jusqu’à 2010. Madsen, Becca; Carroll, Nathaniel; Moore Brands, Kelly; 2010. State of Biodiversity Markets Report: Offset and Compensation Programs Worldwide. Disponible en: http://www.ecosystemmarketplace. com/documents/acrobat/sbdmr.pdf, p. 09. Accédé en 02.03.2012.

24 CDC Biodiversité. Projets. Disponible en: http://www.cdc-biodiversite.fr/content/nosprojets. Accédé en 02.03.2012.

25 La Réserve Légale est le pourcentage d'une propriété, donné par le Code Forestier brésilien, qui limite les droits d'usage du propriétaire, à cause de ses valeurs écologiques. Il ne peut pas utiliser ce pourcetage. (article 16 du Code). L'Aire de Preservation Permanente consiste en une autre sorte de protection légale définie par la loi pour certaines parties spécifiques d'une propriété comme les ressources d'eau, les forêts riveraines, etc. (article 2o du Code). D'ailleurs, le code est en changement dans ce moment, ces pourcentages et l'encadrement de ces endroits peuvent changer encore.

26 Les cas où il y a des activités pour ces marchés sont des projets pilotes dans les villes: Santarém (Pará), Vale do Rio São Lourenço (Mato Grosso), Lucas do Rio Verde (Mato Grosso), Angélica (Mato Grosso do Sul) Guarapuava (Paraná), Sorriso (Mato Grosso), Taguatinha (Tocantins), Tombador Veadeiros (Goiás), Oeste da Bahia (Bahia) e Alto do Itajaí (Santa Catarina), Paragominas. The Nature Conservancy, Calendário de atividades, 2010; et Giordano, Samuel Ribeiro. Calenan, Silvia Morais de Queiroz. Estudo de Caso: 
prix est fait par contrat et sur la base d'un coût d'opportunité, calculé par l'hypothèse de l'utilisation de ces parties des actifs pour d'autres activités. ${ }^{27}$ Inspiré par ce mouvement privé, l'État est devenu plus actif. Il avait quelques programmes locaux, ${ }^{28}$ mais son engagement plus expressif était avec la Mesure Provisoire n.2166-67/2001 pour instaurer un système d'information national sur des crédits et débits, la «Servidão Florestal» et les Quotas de Réserves Forestières, pour représenter la «Servidão». Malgré des défis pour l'effectivité29 des mécanismes de compensation au Brésil, c'est un exemple qui illustre des engagements publics et privés pour l'environnement en utilisant des outils juridiques et économiques.

En Chine, la compensation existe en tant que paiement pour des services environnementaux. Ces programmes sont étatiques et existent depuis 1999. Il y en a pour les forêts, pour des objectifs de lutte contre la désertification et la conservation de l'eau. Ce sont des contrats entre l'État et les agriculteurs pour des actions de restaurations des terres, ou de conservation de l'eau, entre autres. Des exemples peuvent être le «Central Government Forest Ecosystem Compensation Fund (FECF)》, «Natural Forest Protection Program», le «Jiulong River Watershed Eco-compensation», ${ }^{30}$ le «The Conversion of Cropland to Forest and Grassland Program», ${ }^{31}$ entre autres.

preservação ambiental via coordenação de ações. PENSA - Centro de Conhecimento em Agronegócios.

27 Giordano, Samuel Ribeiro. Calenan, Silvia Morais de Queiroz. Estudo de Caso: preservação ambiental via coordenação de ações. PENSA - Centro de Conhecimento em Agronegócios.

28 Les prècedents du CRF concernent le Système de Gestion, Récuperation et Protection de la Réserve Légale et les Aires de Protection Permanente (SISLEG), en 1999, donné par le gouvernenment local du Paraná, et le Système de licences environnementales pour les propriétés rurales (SLAPR), donné par le gouvernement local du Mato Grosso, en 2000. Ils sont des exemples différents de ces de l'activité de la TNC. Schneider, Robert R. What we can learn from SLAPR, SISLEG and CRF. The Nature Conservancy.

29 Ce sont des questions d'insécurité juridiques concernant le changement du Code Forestier, sur l'objet du marché (CRF) et sur des informations sur la qualité de l'environnement, entre autres.

30 Bennet, Michael T. Markets for Ecosystem Services in China: An Exploration of China's "Eco-compensation" and Other Market-Based Environmental Policies. Forest Trends, 2009 , p. 43.

31 Zhang, Qingfeng et al. An eco compensation policy framework for the People's Republic of China: challenges and opportunities. Mandaluyong City, Philippines: Asian Development Bank, 2010, p.35. 
Par ces différents exemples, il est construite une exigence de clarifier quelques enjeux en termes d'outils de théorie du droit, pour l'encadrement de la compensation en tant qu'un objet juridique. Ce sont des questions de pluralité pour ce qui concerne les acteurs et la pluralité d'enjeux touchés par les normes juridiques.

\section{Des questions de pluralité d'acteurs et de pluralité d'enjeux touchés par les normes en face des exemples de compensation}

Des questions de pluralité d'acteurs concernent l'élargissement du concept de droit comme étant toujours un domaine de droit de l'État. Les concepts de pluralisme juridique, corégulation et autorégulation peuvent aider dans cet élargissement (2.1). D'ailleurs, les cas inspirent aussi des questions concernant la pluralité d'enjeux touchés par la norme, dont l'analyse économique du droit de l'environnement et son rapport systémique est importante (2.2).

\subsection{Les concepts de pluralisme juridique, corégulation et autorégulation face aux exemples de compensation}

La caractérisation d'un pluralisme juridique dans la protection de l'environnement existe face à l'ensemble des cas de compensation. Ces mouvements peuvent varier pour ce qui concerne la participation privée, entre les mouvements de corégulation et d'autorégulation. Neanmoins, l'État demeure encore un acteur important. Il faut juste tenir compte que la participation privée peut intégrer les sources juridiques environnementales, en exigeant une adaptation épistémologique de la façon d'encadrer les réseaux juridiques. Car, considérant les exemples de compensation ci-dessus mentionés, la protection environnementale ne se trouve plus seulement dans la norme étatique conventionnelle ou dans l'obligation juridique concernée, il y existe des constructions juridiques hybrides, publiques et privées suscitant la présence d'un pluralisme juridique, de la corégulation et de l'autorégulation pour identifier la forme juridique du réseau normatif apportant une règle de compensation.

Ainsi, il est possible de parler d'un pluralisme juridique aussi dans la protection juridique de l'environnement. Par exemple, dans les banques de compensation aux États-Unis sont le résultat d'une incitation bien adaptée et consistent dans des réseaux normatifs publics et privés pour intégrer l'obligation de compenser à l'offre de crédits qui répresentent la mesure 
compensatoire. Ces crédits sont produits par des tiers, qui s'organisent dans les actions de création, de conservation et de recomposition de la nature. L'incitation concerne l'action étatique de prévoir, ensemble avec l'obligation de compenser, la possibilité de la faire au travers le marché.

Dans le cas américain, le maître d'ouvrage peut compenser lui-même, ou il peut acheter un crédit qui représente cette compensation. La demande pour ces crédits est l'obligation de compenser. Cela stimule le cadre privé à s'organiser pour produire l'offre de cette demande. De cette façon, appuyé par des régulations américaines bien détaillées, le cadre privé s'organise pour constituer le réseau de ce marché. L'institutionnalisation de ces réseaux est si forte qu'il y existe, par exemple, une association industrielle pour les systèmes de banking (des banques de compensation), ainsi qu'une conférence annuelle. D'autres programmes sont le système national de crédit pour des actions de restauration («National Recovery Credit System»), d'autres mécanismes locaux, comme le "Acres for America", le programme volontaire dirigé par Walmart, ou encore le «Bureau of Land Management Offsite Mitigation Policy». ${ }^{32}$

L'argument renforce un retour de la démarche pluraliste en théorie du droit. ${ }^{33}$ Il s'agit d'un retour lorsque le pluralisme juridique n'est pas un concept tellement nouveau. ${ }^{34}$ Toutefois, ici, il réside dans des efforts pour aller au-delà des approches concernant la diversité entre le droit étatique et les divers droits éthiques, culturels, de différentes classes sociales, etc. C'est une approche pluraliste renforcée par des enjeux de la mondialisation et tenue par l'interaction entre l'État et la société civile, dont les acteurs économiques. ${ }^{35}$

Dans ce cadre pluraliste, pour l'encadrement des sources juridiques, nous pouvons utiliser la dénomination «centre/périphérie» ${ }^{36}$ pour

32 Madsen, Becca; Carroll, Nathaniel; Moore Brands, Kelly, "State of Biodiversity Markets Report: Offset and Compensation Programs Worldwide", 2010, Disponible sur http://www. ecosystemmarketplace. com/documents/acrobat/sbdmr.pdf.

33 Ost, F. e Kerchove, De la pyramide au reseau. Pour une théorie dialectique du droit. Bruxelles: Publications des Facultés universitaires Saint-Louis Boulevard du Jardin Botanique, 2002, p.186.

34 Santos, Boaventura de Sousa. Vers un nouveau sens commun juridique. Droit, science et politique dans la transition paradigmatique. Traduction de Nathalie Gonzales Lajoie. Paris, LGDJ, 2004, p.147.

35 Teubner, G. Global Bukowina. In Gunther Teubner (ed.). Global law without a State. Brookfield: Dartmouth, 1997, p. 3-28, p.5.

36 Teubner, Gunter. Breaking frames: Economic globalisation and the emergence of Lex mercatoria. European Journal of Social Theory 5, 2002, 199-217. 
comprendre la décentralisation de la source du droit de l'État. Les forces privées qui résultent en normes, comme celles de la société civile, des acteurs économiques, sont parties des sources périphériques du droit.

Tant dans la France qu'au Brésil, des mouvements similaires commencent à ce former. Il est possible de constater des négociations établies pour la conservation de la nature, à fin d'achever l'obligation de compenser. La participation privée peut être dans la production de la norme, comme au Brésil, aux États-Unis et en France, par des contrats et des autres réseaux normatifs basés sur des réglementations et sur des intérêts privés.

En encadrant des forces publiques et privées dans la constitution des normes, il est possible de vérifier ces réseaux normatifs comme des objets d'une recherche juridique. Les concepts de corégulation et d'autorégulation peuvent aussi aider dans l'analyse de ces sources périphériques. Dans les mots d'Apolline Roger, la corégulation c'est «une intervention normative conjointe des autorités publiques et des destinataires privés dans laquelle chacune des parties conserve un véto sur la norme finale». ${ }^{37}$ Elle se distingue de la réglementation lorsqu'il ne s'agit pas seulement des normes décidées par les autorités publiques. La corégulation c'est une interaction entre l'inaliénabilité des pouvoirs de police de l'administration pour des enjeux environnementaux et la nécessité de renforcer l'engagement privé dans ces enjeux. ${ }^{38}$

L'autorégulation, à la fois, se distingue par l'engagement unilatéral privé. C'est ce que nous avons vu dans les premiers mouvements brésiliens de compensation avec la TNC. D'ailleurs, ce même cas a montré l'engagement public postérieur encadrant la corégulation. Par les cas de compensation, donc, nous pouvons affirmer un pluralisme juridique et l'émergence plutôt de la corégulation, mais sans exclure la possibilité de l'autorégulation.

Ces efforts d'affirmation de l'existence de la corégulation et d'autorégulation, toutefois, ont leurs propres défis. Lorsque la corégulation est évoquée pour expliquer une interaction publique privée qui, en principe, ne consiste pas en l'intervention de l'État pour des objectifs d'ordre public; dans la pratique, toutefois, cela peut être vague, au moins dans les cas d'intérêt

37 Roger, Apolline. Corégulation et Politique climatique de l’Union Européene. Le rôle des accords environnementaux. In: Maljean-Dubois, Sandrine; Roger, Appolline. L'implication des enterprises dans les politiques climatiques. Entre corégulation et autorégulation. CERIC, France, 2011, p. 67.

38 Wemaere, Matthieu; Maljean-Dubois, Sandrine. Introduction générale. In: Maljean-Dubois, Sandrine; Roger, Appolline. L'implication des enterprises dans les politiques climatiques. Entre corégulation et autorégulation. CERIC, France, 2011, p. 21. 
public. ${ }^{39}$ D'ailleurs, il est possible que la caractérisation de l'intérêt public ne soit pas toujours l'intervention de l'État, elle peut venir d'une corégulation. Parce qu'il est difficile de contrôler tous les acteurs privés, pour toutes ces caractéristiques de la mondialisation, de transnationalité, transterritorialité, etc., ${ }^{40}$ il est important d'utiliser des approches qui incitent des actions privées pour compter l'environnement.

Toutefois, l'État joue encore un rôle important dans la protection environnementale. En Chine et dans des compensations des outils juridiques «traditionnels», cependant, l'État est le principal acteur. Les REDDs sont des compensations «transnationales». La Commission de Compensation des Nations Unies n'encadre pas une production juridique conjointe, mais c'est un exemple d'indemnisation environnementale à des acteurs privés en droit international public, en étant une avance en termes de responsabilité environnementale, même dans le cas précis des dommages de guerre. L'MDP, à la fois, caractérise une coopération transnationale, où la participation privée n'est pas limitée au territoire.

En Chine, des politiques pour l'engagement privé dans la protection de la nature existe par des programmes de paiement pour les services environnementaux. Ces programmes sont, par ailleurs, étatiques. Ils ont été inspirés par la nécessité d'améliorer la protection de la nature pour renforcer les vulnérabilités des villages aux risques environnementaux catastrophiques. ${ }^{41}$ Ainsi des paiements ont était fait pour la protection des rivières, car des inondations étaient un gros problème à la société. En autre, des paiements pour la reforestation étaient encadrés, car la dégradation des endroits augmentait la vulnérabilité de l'endroit, entre autres situations. Les paiements pour les services environnementaux sont une des principales stratégies pour la prévention des catastrophes. ${ }^{42}$

39 Delmas-Marty, Mireille. Les forces imaginantes du droit. Le relatif et l'universel. Paris, Éditions du Seuil, p. 2004, p. 330.

40 Frydman, Benoit. Coregulation: A Possible Legal Model for Global Governance January 5, 2012). About Globalization, Views on The Trajectory of Mondialisation, pp. 227-242, B. De Schutter, J. Pas, eds., Brussels, vuB Brussels University Press, 2004. Available at SSRn: http://ssrn. com/abstract=1980176. Accédé en 02.03.2012.

41 UNEP. «Les catastrophes ne sont pas aléatoires et ne se produisent pas par accident. Elles sont la convergence des dangers et des conditions de vulnérabilité.» Environment and Disaster Risk. Emerging Perspectives. Seconde édition July, 2008, p. 9.

42 Carvalho, Délton Winter de. Por uma necessária introdução ao direito dos desastres ambientais. Revista de Direito Ambiental, 67, p. 107-145, 2012. 
Malgré les spécificités de chaque cas, le point à remarquer c'est que la participation privée dans la production et la mise en oeuvre des normes existe. Les critères classiques comme la souveraineté et la contrainte de l'État, ${ }^{43}$ ne sont plus les éléments pour la définition d'un ordre juridique, ni dans le cadre de la protection de l'environnement. La mondialisation cause la relativisation du pouvoir étatique, en inspirant la création d'ordres juridiques venus de la société civile ${ }^{44}$ qui commence à régler ses propres objets et intérêts, dans la mesure où elle peut le faire.

Pour ce qui concerne le défi épistémologique d'aller au-delà de la vision étatique du droit de l'environnement, l'argument impose quelques limites. Ce sont des limites tenues à cause des tensions entre les ordres étatiques et non- étatiques. Ces tensions existent dans la mesure où nous parlons de la protection de la nature, cadre où l'État est présent, tôt ou tard. De plus, en tant qu'une mobilité à faveur de la protection de l'environnement, il n'y a pas encore un mouvement transnational tellement expressif, car la plupart des exemples de participation privée étaient dans le domaine national. L'exemple du MDL et la logique des REDDs, toutefois, nous donnent l'espoir de la possibilité. Ces limites existent, donc, parce que l'État est un acteur encore important dans la protection juridique de l'environnement. Cette importance de l'État, toutefois, n'élimine pas la possibilité d'un pluralisme juridique. Il s'agit, en effet, de renforcer le rôle de tous les acteurs en société publique et privée, pour l'environnement.

Ce sont des efforts qui associent des mesures législatives à des mesures prises par les acteurs privés, où il existe aussi un jeu de forces, un jeu d'intérêt. Car c'est ainsi une question de pouvoir et de vouloir qui encadre des démarches pluralistes. Ainsi, lorsque ces démarches ne sont pas forcement limité par le droit étatique, l'incitation vers des démarches pluralistes «justes» est importante, avec un équilibre entre les enjeux économiques et environnementaux. De plus, au moins pour ce qui concerne l'environnement, pour

43 Comme, par exemple, le concept de droit selon Kelsen: «...La norme juridique est la règle en vertu de laquelle s'opère l'imputation à l'État, qui, en tant que sujet des actes étatiques, n'est que la personnification de l'ordre juridique». Kelsen, Hans. Les rapports de systême entre le droit interne et le droit international public. The Hague Academy of International Law, p. 242-243.

44 Le concept de société civile caractérise l'organisation autour des décisions, comme dans la société publique, mais avec autres acteurs que l'État. C'est une expression qui fait référence à des autres pouvoirs, comme le pouvoir économique ou celui des organisations non-gouvernementales. Delmas-Marty, M. Les forces imaginantes du droit (III) LA refondation des pouvoirs. Editions du seuil, 2007, p. 24. 
que ces mouvements ne finissent pas dans un jeu à suivre le pouvoir du plus fort économiquement, un double renforcement est essentiel: des acteurs autour de l'objet (environnement), de l'importance de l'objet pour les acteurs.

Il faut encadrer théoriquement les concepts de corégulation de l'autorégulation, du pluralisme juridique aussi dans la théorie du droit de l'environnement, à fin d'améliorer l'analyse de l'efficacité juridique environnementale dans un monde globalisé. Les formes de compensation en sont un exemple: la protection environnementale est encadrée tant par les compensations traditionnelles, que par l'incitation du comportement privé. Ainsi, les compensations traditionnelles sont également importantes que celles innovatrices, car ce sont des situations juridiques différentes.

Il s'agit de renforcer l'État et l'action privée dans l'interaction des intérêts publics avec les intérêts privés. C'est pour ce double renforcement que l'interaction entre les instruments juridiques et économiques, ainsi que l'analyse économique du droit de l'environnement sont des outils importants, lorsqu'ils peuvent être une meilleure compréhension et application de l'interaction des différents enjeux économiques et écologiques, dans des normes.

\subsection{L'émergence de l'analyse économique du droit de l'environnement et son rapport systémique en face des exemples de compensation}

L'émergence de l'analyse économique du droit de l'environnement se caractérise face à la pluralité d'enjeux touchés par la norme juridique, surtout à cause de l'importance de l'encadrement du concept d'efficacité économique dans la protection juridique de l'environnement, que nous avons vu avec les mécanismes de compensations. Le rapport systémique ${ }^{45}$ est aussi vu dans l'insertion de cette efficacité économique, et dans des interprétations qui intègrent des concepts économiques dans le langage de la protection juridique de la nature.

Dans tous les exemples, nous avons vu des différentes formes d'intégration des enjeux économiques et écologiques. Nous avons vu des

45 Même si l'étude ne consiste pas en une étude purement «Luhmaniènne»; son concept de système sert comme base dans ce regard systémique de l'interaction entre les différents systèmes sociaux. Luhmann, Niklas. Prefacio a la primera edición Alemana. In: Luhmann, Niklas. Sistemas sociales: Lineamientos para uma teoría general. trad. Silvia Pappe y Brunhilde Erker. Barcelona: Anthropos, 1998, p. 9. 
mesures positives pour l'équilibre dans l'interaction entre des enjeux économiques et écologiques, surtout à cause des encadrements des paiements pour des actions de conservations et restaurations d'espaces naturels. Les différents usages de la logique d'échange vus dans les cas brésilien, américain, français et chinois le montrent. Nous pouvons voir la pertinence des mécanismes de compensation pour une meilleure définition des droits, des échanges et des encadrements des coûts de l'environnement.

Dans ce sens, la compensation par l'interaction avec des outils de marché a amené à une logique économique de prise en compte des conséquences d'un choix dans le milieu par une meilleure définition de leurs coûts, et non seulement par la prise en compte de ces conséquences, comme les outils traditionnels juridiques le font. Ces mécanismes sont des approches incitatives pour le changement des comportements des acteurs économiques, en général.

D'abord, il faut expliquer que, penser sur comment inciter des changements des acteurs économiques est lié à l'analyse économique du droit de l'environnement; ${ }^{46}$ c'est une forme spécifique d'interprétation du droit. ${ }^{47}$ Parce que ce champ d'études est tellement vaste, ${ }^{48}$ nous n'allons voir que la relation de la compensation avec l'insertion de l'efficacité économique selon Kaldor-Hicks, dans la protection juridique de l'environnement.

Dans un sens plus large, les conséquences environnementales négatives d'une activité sont liées aux formes de correction des défaillances du marché, point de vue introduit par l'économie du bien-être, de Pigou. ${ }^{49} \mathrm{Ju}$ ridiquement, c'est la gestion de la pollution, le contrôle des effets de l'action humaine dans le milieu naturel, donné par divers instruments juridiques et économiques encadrés dans des efforts de mise en oeuvre du développement durable.

46 L'internationalisation des externalités est, en effet, la justification première de toute prise en compte des facteurs environnementales par les agentes économiques. Prieur, Michel. Préface. Faure, Michael. L'analyse économique du droit de l'environnement. Belgique: Bruylant Bruxelles, 2007, p. V.

47 Deffains, Bruno; Obidzinski, Marie. Vers de nouveaux questionnements: production; design et évolution des systèmes juridiques, in Deffains, Bruno; Langlais, E. Analyse economique du droit. France: Boeck Universités, 2010, p. 383.

48 Mackaay, Ejan; Rousseau, Stéphane. Analyse Économique du Droit. 2a édition. Paris: éditions Dalloz, 2008, p. 9.

49 Crozet, Y. Analyse économique de l'Etat, Armand Colin/Masson, Paris, 1997, p. 191. 
En analysant le statut de l'efficacité économique par le droit, il y a un rôle de légitimation et justification des normes, quand il s'agit d'importer ce concept dans les analyses juridiques. En effet, le point à souligner ici c'est que la compensation intègre l'efficience économique dans le droit lorsqu'elle peut être vue comme une traduction de l'efficience économique selon KaldorHicks, qui explique qu'un acte sera efficient économiquement quand les gains sont supérieurs aux pertes. ${ }^{50}$ Compenser les dommages environnementaux face à l'intervention de la nature est une telle forme de balance.

C'est la logique du principe «pas de perte nette», caractéristique d'additionnalité de la compensation présente, non seulement dans ce principe, mais dans la caractérisation des actions dites «nécessaires» et pour les buts «d'intérêt général». Dans ce sens large, l'insertion de l'efficience économique existe dans la plupart des mécanismes de compensation, lorsque nous parlons des justificatifs de pertes à cause des gains des interventions dans la nature. Juste des exemples d'indemnisation ne semblent pas encadrer une telle logique, lorsque ce sont des compensations à cause des pertes causées des formes injustes.

De plus, la compensation concerne un regard systémique en appliquant une interprétation économique des enjeux écologiques. Avec des limites imposées de formes normatives, il est possible d'équilibrer cette interprétation avec les enjeux écologiques. Ces limites sont données non seulement par les parties, mais par les logiques de chaque enjeu: la logique des enjeux économiques, de coût des actions, par exemple, avec la logique de la protection de l'environnement. Dans ce sens, l'interaction entre les enjeux est limitée aussi dans la logique de chaque enjeu, dans un mouvement dynamisé par ses tensions.

Cette interaction systémique est aussi présente dans l'utilisation des concepts comme des paiements pour des «services écologiques» etc., compris dans quelques mécanismes de compensation comme dans l'exemple chinois. L'interprétation économique de ces concepts amène à la compréhension des enjeux écologiques et permet de les encadrer dans un langage de coût, plutôt que seulement un langage éthique ou moral, de droits et devoirs, ou de l'intervention de l'État, typique de la logique de la protection environnementale.

50 Pérès, Cécile. Rapport introductif. In: Bollée, Sylvain; Laithier, Yves-Marie; Pérès, Cécile (dir.). L'efficacité économique en droit. Paris: Economica, 2010, p. 09. 
Ainsi, dans la logique de cette analyse, il est possible de mieux travailler l'attribution des droits de propriété et la mise en place des partenariats publicprivés, qui sont des éléments qui renforcent la protection de l'environnement dans un cadre pluraliste. Pour ce double renforcement, donc, l'interaction entre les outils juridiques et économiques est importante, parce qu'ils encadrent des engagements publics et privés et parce qu'ils permettent une meilleure interaction entre les enjeux économiques et écologiques à travers les normes. Cette meilleure interaction, à la fois, est liée au renforcement de la responsabilité des acteurs privés, lorsque ce sont des voies incitatives pour le changement de leurs comportements.

\section{Conclusion}

L'ensemble des cas de compensation nous permet de vérifier quelques-unes des limites et possibilités pour l'épistémologie juridique pour ce qui concerne analyser la protection juridique de l'environnement dans une perspective qui va au-delà de celle traditionnelle du droit étatique qu'englobe une obligation juridique et sa sanction.

D'abord, la caractérisation d'un pluralisme juridique dans la protection de l'environnement existe dans la mesure où il émerge des réseaux normatifs autres que celui étatique. C'est du pluralisme juridique dans une approche moins ou plus limitée puisqu'il est encadré dans des mouvements de corégulation, pourtant sans exclure l'autorégulation. Ce sont des limites tenues à cause des tensions entre les ordres étatiques et non étatiques. Des tensions existantes lorsque l'État est encore un acteur important pour la protection de l'environnement. En même temps, il s'agit de renforcer le rôle de tous les acteurs en société, publics et privés, pour l'environnement. En effet, ce sont des limites et des possibilités de la mondialisation, avec la relativisation du pouvoir étatique.

Pour le double renforcement des rôles publics et privés en temps de mondialisation, l'interaction entre différents enjeux doit être toujours en question dans des normes juridiques. Les mécanismes de compensation ayant des paiements pour des actions de restauration et conservation ont montré que les outils économiques jouent un rôle important dans cette interaction. C'est par cette interaction que nous pouvons voir émerger de différents réseaux normatifs de corégulations et d'autorégulation. Cela montre l'importance de regarder le droit de forme plus systémique par rapport aux enjeux qu'il touche, et la pertinence des études d'analyse économique du droit de l'environnement. 
Les types traditionnels de compensation, qui sont l'indemnisation, la réglementation au sein des études d'impact, les compensations au sein des manques de due diligence en droit international sont importantes dans l'imposition du comportement conventionné entre les États, en droit international, ainsi que dans la relation entre l'État et le citoyen, en droit national. Les incitations par des outils économiques sont également importantes pour l'existence des corégulations et d'autorégulations qui renforcent l'efficacité juridique environnementale par une augmentation de la participation privée dans la protection de la nature.

Ainsi, en affirmant un pluralisme juridique aussi dans le cadre de l'environnement, nous nous dissocions de l'importance de lier le droit à l'État pour le tenir juste comme un instrument pour des fins d'organisation des enjeux en société. Nous déplaçons le regard juridique pour aussi prendre en compte des influences clés dans le changement et constitution des normes. Une recherche dans ce sens concerne plus les enjeux d'effectivité. Les divisions épistémologiques d'ordre étatique/non-étatique, public/privé, international/national sont utiles juste pour l'identification des éléments présents dans l'analyse: dans quel cadre la norme a été formée, comment elle a été formée, etc. C'est, donc une division pédagogique générale.

De plus, l'analyse économique du droit est importante dans la mesure où elle peut aider dans l'interprétation des normes juridiques. Dans le cas de la compensation en droit, elle est essentielle justement, car elle aide à comprendre la logique de la compensation comme étant un outil de balance entre les gains et les pertes d'une relation. Il est une forme juridique d'internaliser l'externalité environnementale négative: on compense les pertes occasionnées d'un dommage environnemental, d'une activité économique.

Ainsi, par les cas de compensation, le pluralisme juridique, la corégulation, l'autorégulation et l'analyse économique du droit sont des outils utiles pour l'analyse du droit de l'environnement au sein de la mondialisation. C'est à l'enseignement juridique de clarifier ces enjeux possibles pour le droit dans un cadre pluraliste, de pluralité d'acteurs, d'enjeux, etc.

\section{Références bibliographiques}

Arkkainen, B.; Radley C., "Information-forcing environmental regulation", Florida State University Law Review, Vol. 33:861, 2006.

Bennett, Michael T. Markets for Ecosystem Services in China: An Exploration of China's "Eco-compensation" and Other Market-Based Environmental Policies. Forest Trends, 2009. 
Bilder, Richard. Settlement of Disputes in Field of International Law of Environment. The Hague Academy of International Law

Cafaggi, Fabrizio, «New Foundations of Transnational Private Regulation», Journal of Law and Society, Volume 38, Number 1, March, 2011.

CDC Biodiversité. Projets. Disponible en: < http:/ / www.cdc-biodiversite.fr/ content/nos-projets. Accédé en 02.03.2012>.

Convention de Bâle sur le contrôle des mouvements transfrontaliers de déchets dangereux.

Convention on the Law of the Non-navigational Uses of International Watercourses1. New York, 21 May 1997.

Deffains, Bruno; Obidzinski, Marie. Vers de nouveaux questionnements: production; design et évolution des systèmes juridiques, in Deffains, Bruno; Langlais, E. Analyse Economique du Droit. France: Boeck Universités, 2010

Delmas-Marty, Mireille. Les forces imaginantes du droit (III) La refondation des pouvoirs. Editions du seuil, 2007.

Delmas-Marty, Mireille. Les forces imaginantes du droit. Le relatif et l'universel. Paris, Éditions du Seuil, p. 2004.

Carvalho, Délton Winter de. Por uma necessária introdução ao direito dos desastres ambientais. Revista de Direito Ambiental, 67, p. 107-145, 2012.

Frydman, Benoit. Coregulation: A Possible Legal Model for Global Governance (January 5, 2012). About Globalization, Views On The Trajectory Of Mondialisation, pp. 227-242, B. De Schutter, J. Pas, eds., Brussels, vuB Brussels University Press, 2004. Available at SSRN: < http://ssrn. com $/$ abstract $=1980176>$. Accédé en 02.03.2012.

Giordano, Samuel Ribeiro. Calenan, Silvia Morais de Queiroz. Estudo de Caso: preservação ambiental via coordenação de ações. PENSA - Centro de Conhecimento em Agronegócios.

Harrington, Winston; Morgenstern, Richard D., «Economic Incentives versus Command and Control, what's the best approach for solving environmental problems?», Ressources, Fall/Winter 2004, disponible sur < http://www.rff.org/rff/Documents/RFF_Resources_152_ ecoincentives.pdf $>$, p. 17.

Kelsen, Hans. Les rapports de systême entre le droit interne et le droit international public, Vol. 014 (1926). The Hague Academy of International Law.

Luhmann, Niklas. Sistemas Sociales. Lineamientos para una teoría general. trad. Silvia Pappe y Brunhilde Erker. Barcelona: Anthropos, 1998.

Mackaay, Ejan; Rousseau, Stéphane. Analyse Économique du Droit. 2a édition. Paris: éditions Dalloz, 2008. 
Madsen, Becca; Carroll, Nathaniel; Moore Brands, Kelly, "State of Biodiversity Markets Report: Offset and Compensation Programs Worldwide", 2010, Disponible sur <http://www.ecosystemmarketplace. com/documents/acrobat/sbdmr.pdf $>$.

Martin, Jean-Christophe. The United Nations Compensation Commission Practice with regards to environmental claims. In: Majean-Dubois, Sandrine. The transphormations of international environmental law, 2011.

OCDE. Recommandation C(72)128 de 26 mai 1972.

OCDE. Recommandation [C(90)177/FINAL].

OCDE. Recommandation du Conseil de 21 avril 2004.

Ost, F. e Kerchove, De la pyramide au reseau. Pour une théorie dialectique du droit. Bruxelles: Publications des Facultés universitaires Saint-Louis Boulevard du Jardin Botanique, 2002.

Pérès, Cécile. Rapport introductif. In: Bollée, Sylvain; Laithier, Yves-Marie; Pérès, Cécile (dir.). L'efficacité économique en droit. Paris: Economica, 2010.

Prieur, Michel. Préface. Faure, Michael. L'analyse economique du droit de l'environnement. Belgique: Bruylant Bruxelles, 2007.

Résolution de l'Institut de Droit International, de la Session de Salzbourg 1961, Utilisation des eaux internationales non maritimes. Disponible en: <http://www.idi-iil.org/idif/resolutionsf/1961_salz_01_fr.pdf acesse en 20.12.2011>.

Roger, Apolline. Corégulation et Politique climatique de l'Union Européene. Le rôle des accords environnementaux. In: Maljean-Dubois, Sandrine; Roger, Appolline. L'implication des enterprises dans lespolitiques climatiques. Entre corégulation et autorégulation. CERIC, France, 2011.

Sadeller, Nicolas de, «Les approches volontaires en droit de l'environnement, expression d'un droit post-moderne ?’ In: Hervé-Fournenreau, Nathalie, "Les approches volontaires et le droit de l'environnement», PUR, p. $45-47$

Santos, Boaventura de Sousa. Vers un nouveau sens commun juridique. droit, science etpolitique dans la transition paradigmatique. Traduction de Nathalie Gonzales Lajoie. Paris, LGDJ, 2004.

Schneider, Robert R. What we can learn from Slapr, Sisleg and Crf. The Nature Conservancy.

Session 7a de la Conférence des Parties, San José, Costa Rica, de 10 au 18 mai 1999. Disponible en: <http://www.ramsar.org/cda/fr/ ramsar-documents-resol-resolution-vii-21-21534/main/ramsar/131-107\%5E21534_4000_1_> Acessé: 29.12.2011. 
Tabau, Anne Sophie. Les interactions des contrôles internationaux et communautaires de La mise en oeuvre du Protocole de Kyoto. Thèse pour l'obtention du diplôme de Doctorat de Droit Public. Université de Droit , D'Economie et de Sciences d'Aix-Marseille III.

Teubner, G. Global Bukowina. In Teubner, Gunther (ed.). Global law without a State. Brookfield: Dartmouth, 1997, p. 3-28.

Teubner, Gunter. Breaking frames: Economic globalisation and the emergence of Lex mercatoria. European Journal of Social Theory 5, 2002, 199-217.

Teubner, Gunther, «Droit et réflexivité. L'auto-référence en droit et dans l'organisation»,L.G.D.J-Bruylant, 1996.

The Nature Conservancy, Calendário de atividades, 2010.

The UNCC Secretariat. Introduction. Disponible en: < http://www.uncc.ch/ introduc.htm>. Acéssé en 29.12.2011

The UNCC Secretariat. Décision 07. Disponible en: <http://www.uncc.ch/ decision/dec_07r.pdf>. Acessé en 22.12.2011.

The unCC Secretariat. The Claims. Disponible en: <http://www.uncc.ch/ theclaims.htm>. Acesse en 12.12.2011.

UNEP, Les catastrophes ne sont pas aléatoires et ne se produisent pas par accident. Elles sont la convergence des dangers et des conditions de vulnérabilité, en: Environment and Disaster Risk. Emerging Perspectives. Seconde édition, July 2008, p. 9.

UN-REDD Programme. Disponible en: <http://www.un-redd.org>. Accédé en 02.03.2012.

Wyeth, George B., «»Standard» and «Alternative» Environmental Protection: The Changing Role of Environmental Agencies», William \& Mary Environmental Law and Policy Review Volume 31 | Issue 1, Article 3, 2006, disponible sur <http://scholarship.law.wm.edu/wmelpr/ vol31/iss1/3>, p. 72 .

Wemaere, Matthieu; Maljean-Dubois, Sandrine. Introduction générale. In: Maljean-Dubois, Sandrine; Roger, Appolline. L'implication des enterprises dans les politiques climatiques. Entre corégulation et autorégulation. CERIC, France, 2011.

Y. Crozet, Analyse économique de l'Etat, Armand Colin/Masson, Paris, 1997.

Zhang, Qingfeng et al. An eco compensation policy framework. for the People's Republic of China: challenges and opportunities. Mandaluyong City, Philippines: Asian Development Bank, 2010. 